\title{
Design and Experimental Validation of MIMO Multiuser Detection for Downlink Packet Data
}

\author{
Dragan Samardzija \\ Bell Laboratories, Lucent Technologies, Holmdel, NJ 07733, USA \\ Email:dragan@lucent.com \\ Angel Lozano \\ Bell Laboratories, Lucent Technologies, Holmdel, NJ 07733, USA \\ Email:aloz@lucent.com

\section{Constantinos B. Papadias} \\ Bell Laboratories, Lucent Technologies, Holmdel, NJ 07733, USA \\ Email:papadias@lucent.com
}

Received 8 March 2004; Revised 29 October 2004

\begin{abstract}
In single-user MIMO communication, the first-order throughput scaling is determined by the smallest of the number of transmit and receive antennas. This typically renders terminals the constraining bottleneck. In a multiuser downlink, this bottleneck can be bypassed by having the base station communicate with multiple terminals simultaneously, in which case the receive antennas at those terminals are effectively pooled in terms of the capacity scaling. This, however, requires that the base have instantaneous channel information. Without such information, the structure and statistics of the channel can be exploited to form multiple simultaneous beams towards the various users, but these beams are in general mutually interfering. This paper proposes the use of multiuser detection to discriminate the signals conveyed over interfering beams. This approach is formulated and experimentally evaluated on an HSDPA MIMO testbed that involves a commercial base station, multiantenna terminals, and custom ASICs.
\end{abstract}

Keywords and phrases: MIMO, HSDPA, UMTS, experimental validation.

\section{INTRODUCTION}

MIMO (multiple-input multiple-output) schemes utilizing multiple transmit and receive antennas are posed to be a major ingredient in the evolutionary process of wireless communication. Widely recognized features associated with MIMO are spatial diversity, signal enhancements, interference mitigation, and spatial multiplexing. The latter, in particular, has driven a lot of the research over the last decade, ever since it was shown in $[1,2]$ that-in adequate channel conditions - the ergodic capacity (in bps/ $\mathrm{Hz}$ ) of a MIMO link as function of the average SNR (signal-to-noise ratio) behaves as

$$
C(\mathrm{SNR})=\min \left(n_{\mathrm{T}}, n_{\mathrm{R}}\right) \log _{2} \mathrm{SNR}+O(1),
$$

where $n_{\mathrm{T}}$ and $n_{\mathrm{R}}$ denote the numbers of transmit and receive antennas, respectively. This linear scaling with the number of antennas is a powerful means to achieve high spectral utilization provided that antenna arrays can be effectively deployed.

In actual wireless systems, of course, links do not operate in isolation: each base station must actively communicate with a plurality of users and thus a number of MIMO links have to coexist. The behavior expressed by (1) can be immediately translated onto a multiuser environment by partitioning either time or frequency onto orthogonal sets, each of which is assigned to a particular user link. Focusing on the downlink, where $n_{\mathrm{T}}$ indicates the number of transmit antennas at the base station while $n_{\mathrm{R}}$ represents the number of receive antennas at the terminal, such orthogonal multiplexing incurs only a small loss in capacity if $n_{\mathrm{R}} \gg n_{\mathrm{T}}$. Unfortunately, the small size and cost sensitivity of mobile terminals precludes the deployment of a large number of antennas thereon and hence the most likely scenario for mobile systems corresponds with $n_{\mathrm{T}} \geq n_{\mathrm{R}}$. (In some cases, $n_{\mathrm{R}}$ may be tightly restricted to equal 1.) In these conditions, orthogonal multiplexing severely constrains the capacity.

Without the constraint of time/frequency multiplexing, a downlink with $n_{\mathrm{T}}$ antennas at the base and $n_{\mathrm{R}}$ antennas at each of $K$ users can yield a sum capacity that behaves as

$$
C(\mathrm{SNR})=\min \left(n_{\mathrm{T}}, K n_{\mathrm{R}}\right) \log _{2} \mathrm{SNR}+\mathrm{O}(1),
$$


whereby the tight restrictions on $n_{\mathrm{R}}$ become immaterial and the burden of limiting the capacity shifts to the base station, where $n_{\mathrm{T}}$ can be more easily scaled. Unfortunately, achieving (2) requires that the base station have accurate and instantaneous information about the state of the fading channel between each of its antennas and those at each of the mobiles $[3,4,5,6]$. This represents a total of $n_{\mathrm{T}} \times K \times n_{\mathrm{R}}$ time-varying complex coefficients, whose instantaneous tracking by the base station is not a viable option in frequency-duplexed systems. ${ }^{1}$

Without instantaneous channel state information at the transmitter, simultaneous transmission to multiple users becomes challenging. In these conditions, interestingly, antenna correlation-often detrimental in MIMO-facilitates the formation of beams that can be directed to individual users providing partial isolation between their simultaneous transmissions. Moreover, this approach (already recognized and incorporated into the UMTS evolutionary process $[7,8]$ ) results in simple receiver structures. The degree of user isolation that can be attained through the composition of beams, however, is directly determined by the location of the users in the cell and by the characteristics of their propagation channels. Unless $K \ll n_{\mathrm{T}}$, every beam will often illuminate users other than the intended one resulting in significant levels of multiuser interference.

In this paper, we formulate and experimentally evaluate a scheme that provides resilience to strong multiuser interference when multiple beams are simultaneously active. The cornerstone of this scheme is the recognition that wellknown MUD (multiuser techniques) [9], developed originally for CDMA (code-division multiple access), can be applied to the mitigation and removal of spatial interference. This represents, to some extent, an abandonment of the idea of simple and basically passive terminals and an embracing of the concept of smart terminals that actively participate in the task of discriminating transmissions to the different users. This conceptual shift is grounded on the rapid improvement in processing power that stems from Moore's law.

In the remainder of the paper, we describe the MIMOMUD scheme and we quantify its benefits through a series of experiments executed on a testbed that involves a commercial base station equipped with multiple antennas, terminals also equipped with multiple antennas, and specially designed MIMO ASICs (application-specific integrated circuits). In order to render the experiments specific, the testbed is set up to comply with the HSDPA (high-speed downlink packet access) channel, which is foreseen to become the main vehicle for the provision of packet data in UMTS. To the best of our knowledge, these are the first such reported experiments.

\footnotetext{
${ }^{1}$ In time-duplexed systems, the reciprocity in the channel propagation characteristics makes it feasible to track these coefficients instantaneously as long as the Doppler spread is small enough. Note, however, that reciprocity does not necessarily apply to the transceivers and thus careful calibration may be required. Note also that the wide-area communication marketplace is currently dominated by frequency-duplexed systems.
}

The paper is organized as follows. Having justified the interest in the simultaneous transmission to multiple users through parallel beams, in Section 2, we review several wellknown MUD approaches and discuss their applicability to the problem of mitigating the effects of multiuser interference across beams. In Section 3, in turn, we briefly describe the key features of UMTS HSDPA and describe in detail its implementation on the MIMO testbed platform. Finally, Section 4 lays down a number of experimental results that validate the applicability of the chosen MIMO-MUD approach.

\section{MIMO-MUD: FORMULATION}

Although not a requirement when MUD is used, we will limit the number of active users to be $K \leq n_{\mathrm{T}}$, which allows for the generation of beams that are orthogonal in origin [10]. Larger numbers of users can of course be accommodated via time/frequency multiplexing.

The baseband complex linear model describing the communication between the base station and the $k$ th terminal, $k \in\{1, \ldots, K\}$, is

$$
\mathbf{y}_{k}=\mathbf{H}_{k} \mathbf{x}+\mathbf{n}_{k},
$$

where $\mathbf{y}_{k}$ is the $\left(n_{\mathrm{R}} \times 1\right)$ vector received by terminal $k, \mathbf{n}_{k}$ is the corresponding additive white Gaussian noise vector with one-sided spectral density

$$
N_{0}=\frac{E\left[\|\mathbf{n}\|^{2}\right]}{n_{\mathrm{R}}},
$$

and $\mathbf{H}_{k}$ is the $\left(n_{\mathrm{R}} \times n_{\mathrm{T}}\right)$ channel random matrix whose $(i, j)$ th entry represents the transfer coefficien $t^{2}$ between the $j$ th base transmit antenna and the $i$ th receive antenna at terminal $k$. In turn, $\mathbf{x}$ is the $\left(n_{\mathrm{T}} \times 1\right)$ transmit vector, common to all users and structured as

$$
\mathbf{x}=\sum_{\ell=1}^{K} \mathbf{w}_{\ell} s_{\ell}
$$

where $s_{\ell}$ is the information-bearing signal intended for terminal $\ell$ while the vector $\mathbf{w}_{\ell}$ contains the set of deterministic coefficients that, applied to each of the transmit antennas, generate the corresponding beam. Without loss of generality, the $\mathbf{w}_{\ell}$ 's are chosen such that $\left\|\mathbf{w}_{\ell}\right\|=1, \ell \in\{1, \ldots, K\}$, and the power radiated for user $\ell$ is then $P_{\ell}=E\left[\left|s_{\ell}\right|^{2}\right]$. It is important to point out that, without instantaneous channel information at the transmitter, the coefficients in the set of vectors $\mathbf{w}_{\ell}, \ell \in\{1, \ldots, K\}$, cannot depend on the random matrices $\mathbf{H}_{\ell}, \ell \in\{1, \ldots, K\}$, but only on their distributions.

\footnotetext{
${ }^{2}$ In order to focus on the spatial processing aspects, the channel fading is modeled as frequency flat. The formulation, nonetheless, can be extended to frequency-selective fading.
} 
From the standpoint of user $k$, we can conveniently rearrange (3) and (5) as

$$
\mathbf{y}_{k}=\underbrace{\mathbf{H}_{k} \mathbf{w}_{k} s_{k}}_{\begin{array}{c}
\text { signal intended } \\
\text { for terminal } k
\end{array}}+\underbrace{\sum_{\ell \neq k} \mathbf{H}_{k} \mathbf{w}_{\ell} s_{\ell}}_{\text {interference }}+\underbrace{\mathbf{n}_{k}}_{\text {noise }},
$$

where the interference corresponds to the signals that are being beamed towards terminals other than $k$, orthogonal in origin but-in general-not upon reception because of the random matrix $\mathbf{H}_{k}$ whose realization is unknown to the transmitter. The realization of $\mathbf{H}_{k}$, in contrast, is considered known to the receiver, which may estimate it provided, for example, that each individual beam is associated with a unique pilot. Multiple secondary pilots are already supported in UMTS [8] and are expected to be equally available in future system designs. More specifically, this enables receiver $k$ to estimate the effective channels $\mathbf{H}_{k} \mathbf{w}_{\ell}$ for $\ell \in\{1, \ldots, K\}$.

There are several manners in which the presence of the interference can be addressed.

(i) The simplest approach is to ignore the interference by matching the receiver at terminal $k$ to the effective channel for its desired signal generating the decision statistic $\left(\mathbf{w}_{k}^{\dagger} \mathbf{H}_{k}^{\dagger} \mathbf{y}_{k}\right)$, which exhibits an average signal-to-interferenceand-noise ratio [9]

$$
\begin{aligned}
& \operatorname{SINR}_{k} \\
& =E\left[\frac{P_{k}\left(\mathbf{w}_{k}^{\dagger} \mathbf{H}_{k}^{\dagger} \mathbf{H}_{k} \mathbf{w}_{k}\right)^{2}}{N_{0} \mathbf{w}_{k}^{\dagger} \mathbf{H}_{k}^{\dagger} \mathbf{H}_{k} \mathbf{w}_{k}+\mathbf{w}_{k}^{\dagger} \mathbf{H}_{k}^{\dagger} \mathbf{H}_{k}\left(\sum_{\ell \neq k} P_{\ell} \mathbf{w}_{\ell} \mathbf{w}_{\ell}^{\dagger}\right) \mathbf{H}_{k}^{\dagger} \mathbf{H}_{k} \mathbf{w}_{k}}\right]
\end{aligned}
$$

which depends strongly on the structure of $\mathbf{H}_{k}$. We will use this SUMF (single-user matched-filter) receiver as a baseline for later comparisons.

(ii) A more robust approach consists of mitigating the interference through MMSE (minimum mean-square error) linear processing, which exploits the information provided by the conditional interference covariance

$$
\boldsymbol{\Phi}=N_{0} \mathbf{I}+\sum_{\ell \neq k} P_{\ell} \mathbf{H}_{k} \mathbf{w}_{\ell} \mathbf{w}_{\ell}^{\dagger} \mathbf{H}_{k}^{\dagger}
$$

The resulting average SINR at terminal $k$ is [9]

$$
\mathrm{SINR}_{k}=P_{k} \mathbf{w}_{k}^{\dagger} E\left[\mathbf{H}_{k}^{\dagger} \boldsymbol{\Phi}^{-1} \mathbf{H}_{k}\right] \mathbf{w}_{k}
$$

which must lie within

$$
\frac{P_{k}}{\sum_{\ell \neq k} P_{\ell}} \leq \operatorname{SINR}_{k} \leq \frac{P_{k} \mathbf{w}_{k}^{\dagger} E\left[\mathbf{H}_{k}^{\dagger} \mathbf{H}_{k}\right] \mathbf{w}_{k}}{N_{0}}
$$

The lower bound in (10) corresponds to an interferencelimited situation with $\mathbf{H}_{k}$ having independent entries, in which case the use of beams provides no significant advantage over time/frequency multiplexing. The upper bound, on the other hand, corresponds to a highly structured channel allowing for the formation of beams that remain essentially orthogonal regardless of the realization of $\mathbf{H}_{k}$, in which case terminal $k$ receives no interference from any of the beams directed to other users.

(iii) The most ambitious approach, and the one embraced in the remainder of the paper, is based on the joint detection of the signals transmitted on all beams, of which only the intended one is decoded and passed on to the higher layers while the remaining ones are simply discarded. In this case, the average SNR per receive antenna at terminal $k$ is simply

$$
\begin{aligned}
\mathrm{SNR}_{k} & =\frac{E\left[\left\|\mathbf{H}_{k} \mathbf{x}\right\|^{2}\right]}{E\left[\left\|\mathbf{n}_{k}\right\|^{2}\right]} \\
& =\frac{\sum_{\ell} \mathbf{w}_{\ell}^{\dagger} E\left[\mathbf{H}_{k}^{\dagger} \mathbf{H}_{k}\right] \mathbf{w}_{\ell}}{N_{0} n_{\mathrm{R}}} .
\end{aligned}
$$

More specifically, the MIMO-MUD solution that we propose relies on terminal $k$ using its knowledge of $\mathbf{H}_{k} \mathbf{w}_{\ell}$ for all $\ell$ to perform ML (maximum likelihood) detection as

$$
\left\{\hat{s}_{1}, \ldots, \hat{s}_{K}\right\}=\arg \min \left\|\mathbf{y}_{k}-\mathbf{H}_{k} \sum_{\ell} \mathbf{w}_{\ell} \hat{s}_{\ell}\right\|^{2},
$$

where $\hat{s}_{k}$ is the estimate of the signal $s_{k}$, retained and processed, while $\hat{s}_{\ell}, \ell \neq k$, are the signals intended for other users, discarded after detection.

\section{HIGH-SPEED DOWNLINK PACKET ACCESS MIMO TESTBED}

\subsection{High-speed downlink packet access}

For delay-tolerant data traffic, upcoming releases of UMTS will allocate a fraction of the power and code space to HSDPA, whose main features are the following.

(i) Time multiplexing. Users are time multiplexed in short frames.

(ii) Multicode signaling. The entire HSDPA code space is assigned to the active user. Thus, the transmit signal consists of a superposition of orthogonal codes.

(iii) No power control. Power control is disabled.

(iv) Link adaptation. The transmit rate is adapted based on feedback from the terminals.

(v) Hybrid $A R Q$. The link-layer automatic repeat request (ARQ) mechanism is combined with the physical-layer forward error correction [11].

With the incorporation of MIMO, the possibility of having active users on separate beams is enabled and, correspondingly, the use of MIMO-MUD becomes alluring. In the remaining, we validate this idea using a $5 \mathrm{MHz}$ MIMO testbed that operates at $2.1 \mathrm{GHz}$ and supports $n_{\mathrm{T}}=4$ antennas at the base and $n_{\mathrm{R}}=4$ antennas at each terminal [12]. The testbed is currently compliant with the above-described HSDPA features. 


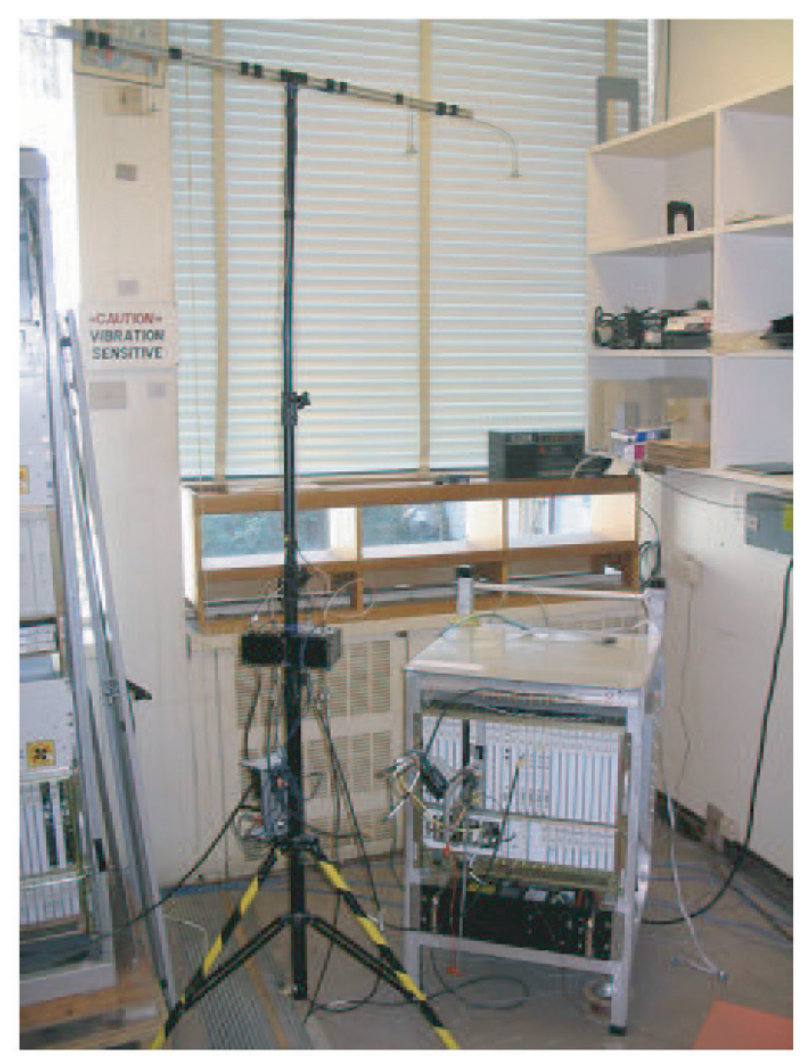

Figure 1: Multiantenna base station.

In order to test the MIMO-MUD concept under the harshest conditions, trivial beams are employed: each $\mathbf{w}_{\ell}$ is identically zero except for the $\ell$ th entry, which is set to 1 . With that, the beams give rise to severe interference as no attempt is made to isolate the transmission to different users.

\subsection{Transmitter implementation}

At the base, omnidirectional vertically polarized 1/4wavelength antennas are set 4 wavelengths apart along a line at a height of about $3 \mathrm{~m}$. As shown in Figure 1, the transmitter is mounted on a prototype Lucent base station (OneBTS ${ }^{\mathrm{TM}}$ prototype). A prototype mezzanine board is used to implement the physical and MAC (medium access control) layers. The rest of the base station, including RF (radio frequency) front end, backplane, and network interface, is also used. The RF front end, in particular, meets the EVM (error vector magnitude) requirements set by Release 5 of the UMTS specifications.

A FPGA (field-programmable gate array) is used to implement the multiantenna physical layer transmitter. The corresponding functional block scheme is depicted in Figure 2 with each functional block being HSDPA compliant. Up to 4 independent data streams $d_{\ell}, \ell \in\{1,2,3,4\}$, are passed down from the MAC layer, each intended for a distinct user. After being independently processed, every stream is radiated out of one of the antennas with a 24- bit CRC (cyclic redundancy code) word appended to each data block. These data blocks are encoded using a rate-1/3 turbo code and the desired transmission data rate is realized via a rate matching procedure that performs either puncturing or repetition of the encoder outputs. Binary words are then mapped to a particular QAM constellation (both QPSK and 16-QAM are supported by HSDPA) and then assigned to specific length16 orthogonal channelization (i.e., spreading) codes. In addition, a unique pilot drawn from a set of secondary UMTS pilots [8] is assigned to each transmit antenna. The pilots are mutually orthogonal and orthogonal to the data-carrying spreading codes. The pilot power is set to $10 \%$ of the total radiated power [13]. The same scrambling code is used at every transmit antenna and the primary and secondary synchronization channels are also transmitted allowing mobile terminals to achieve chip-level, slot-level, and frame-level synchronization and to perform cell search procedures. The above functional blocks (in Figure 2) are implemented on FPGA Xilinx Virtex II 6000, with the clock rate of $61.44 \mathrm{MHz}$ using approximately $15 \%$ of the available logic (i.e., logic slices), for each user (i.e., transmitted stream). Furthermore, approximately $100 \mathrm{kB}$ memory is used per user.

The rate controller in Figure 2 is closely coupled with the multiuser scheduling that is executed at the MAC layer. Specifically, the rate controller is responsible for setting, for each 2-milliseconds time transmission interval, the rate matching parameters, modulation (QPSK or 16-QAM), and number of active spreading codes . Effectively, it optimizes the transmission data rates for a given channel and data traffic conditions. For the experimental results presented in Section 4, QPSK modulation was used with rate-1/2 coding and 10 length-16 active spreading codes.

To support multiple users, the MAC layer is implemented on a processor platform. Specifically, the multiuser scheduling and hybrid-ARQ are implemented on a digital signal processor (Texas Instruments DSP 6701), while interfacing to an IP (Internet Protocol) network is implemented on an embedded processor (Motorola PowerPC 8260). The standard HSDPA specifications are retained at the MAC layer and thus only the physical layer is aware of the presence of MIMO.

\subsection{Receiver implementation}

As shown in Figure 3, the terminal antennas are low-profile bow-tie printed dipoles with alternating $45^{\circ}$ polarizations occupying vertexes of an $8.2^{\prime \prime} \times 5.2^{\prime \prime}$ rectangle with the entire array fitting on the back of a laptop. Note that the fifth antenna, which is placed in the center of the rectangle (in Figure 3), is vertically polarized and is used for the uplink transmission (a conventional single transmit antenna uplink is used). Physically different downlink and uplink antennas are used to simplify the design by avoiding implementation of an analog antenna coupler (which is otherwise needed when the same antenna is used both for the uplink and downlink, simultaneously).

The functional block scheme of the multiantenna physical layer receiver is illustrated in Figure 4, where $\hat{d}_{k}$ is the estimate of the transmitted data for terminal $k$. After the AD (analog-to-digital) conversion, the received signal is sent to the MIMO-MUD ASIC which, in turn, outputs LLRs (loglikelihood ratios) that are then fed to the rate dematcher. 


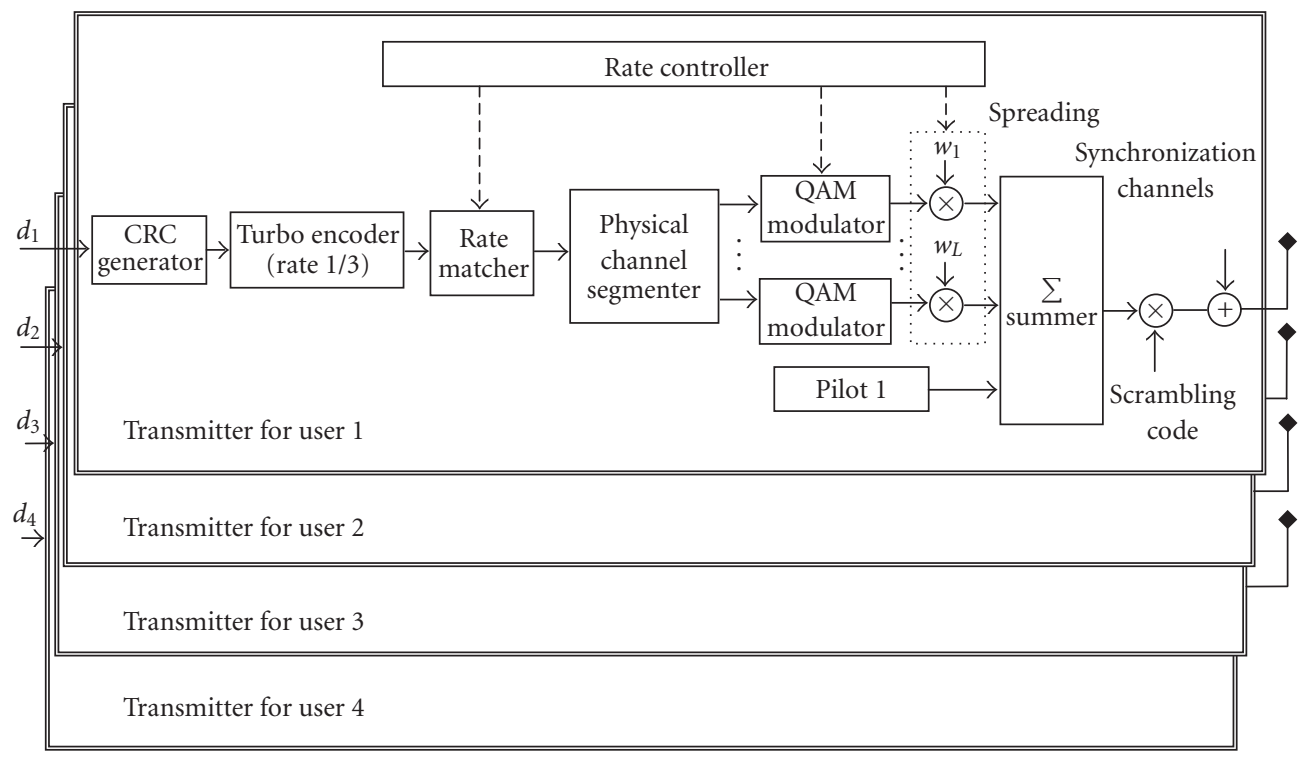

FIGURE 2: Functional block scheme of multiantenna HSDPA physical layer transmitter.

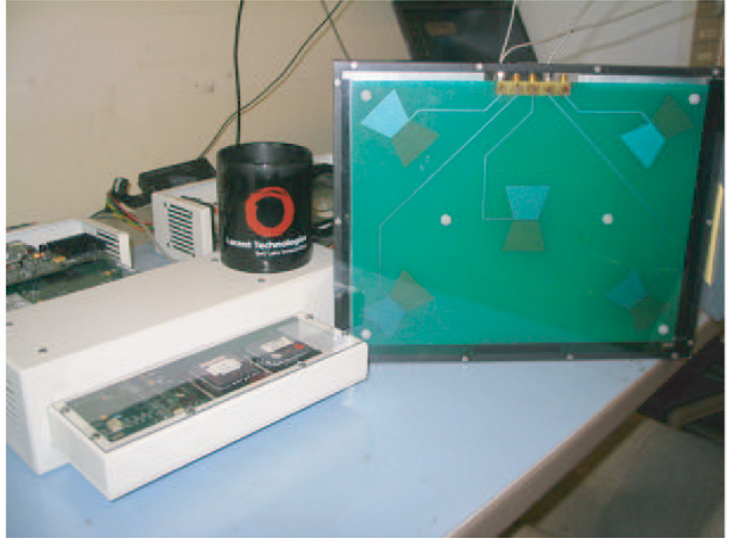

Figure 3: Terminal and receive antenna array.

After dematching, the turbo decoder ASIC performs iterative decoding. The physical layer is implemented on 3 interconnected printed circuit cards that are next described in more detail.

Card 1 implements the analog RF front end outputting up to 4 complex baseband signals, each corresponding to a receive antenna. A heterodyne receiver with a $140 \mathrm{MHz}$ IF and noise figure under $8 \mathrm{~dB}$ is utilized, after which 10 - bit $\mathrm{AD}$ conversion takes place.

Card 2 contains the basic processing elements of the multiantenna receiver: (i) MIMO-MUD ASIC ${ }^{3}$ [14] and (ii) turbo decoder ASIC [15]. A block scheme of the MIMO detector is given in Figure 5. The detector is based on a bank of

\footnotetext{
${ }^{3}$ The MIMO-MUD ASIC is manufactured using 0.18-micron CMOS technology, with 438000 gates, $300 \mathrm{~mW}$ core power, and size of $3.7 \mathrm{~mm} \times$ $3.7 \mathrm{~mm}$.
}

despreaders matched to the data-carrying spreading codes, whose outputs are fed into the ML detection that corresponds with (12). In Figure 5, $\hat{s}_{l k}, l \in\{1, \ldots, L\}$, is the estimate of the transmitted symbol corresponding to code $l$ for user $k$. Furthermore, each LLR corresponds to 1 channel bit with an 8 - bit resolution.

An estimate of the MIMO channel, essential to the detection process, is obtained from an on-chip estimator. This is illustrated in Figure 6, where $\underline{h}_{i j}$ denotes the estimate of the $(i, j)$ th entry of the MIMO channel matrix $\mathbf{H}_{k}$. The on-chip estimator is based on a bank of despreaders corresponding to each of the length-512 pilot codes. In the case of frequencyflat fading, the presented estimator results in an ML channel estimate (see [13] and references therein). To lower the estimation noise, an optional integrator with forgetting factor $\alpha$ is available. For the experimental results in Section $4, \alpha=0$.

Card 3, finally, holds the FPGA that acts as interconnect matrix between ADs, MIMO-MUD ASIC, and turbo decoder ASIC. Furthermore, it executes (i) synchronization, (ii) frequency offset compensation, (iii) physical channel desegmentation, (iv) rate dematching, (v) CRC check, and (vi) numerous auxiliary functions. To all of these functions, the use of MIMO is immaterial. The above functions are implemented on FPGA Xilinx Virtex II 6000, with the clock rate of $61.44 \mathrm{MHz}$ using approximately $25 \%$ of the available logic and $70 \mathrm{kB}$ of memory.

\section{MIMO-MUD EXPERIMENTAL RESULTS}

Indoor over-the-air measurements, mostly in static conditions, were carried out in a laboratory/office environment. The receiver was placed at various locations in the room. QPSK modulation was used with rate-1/2 coding and 10 length- 16 orthogonal codes. The measurements include thermal as well as quantization noise. 


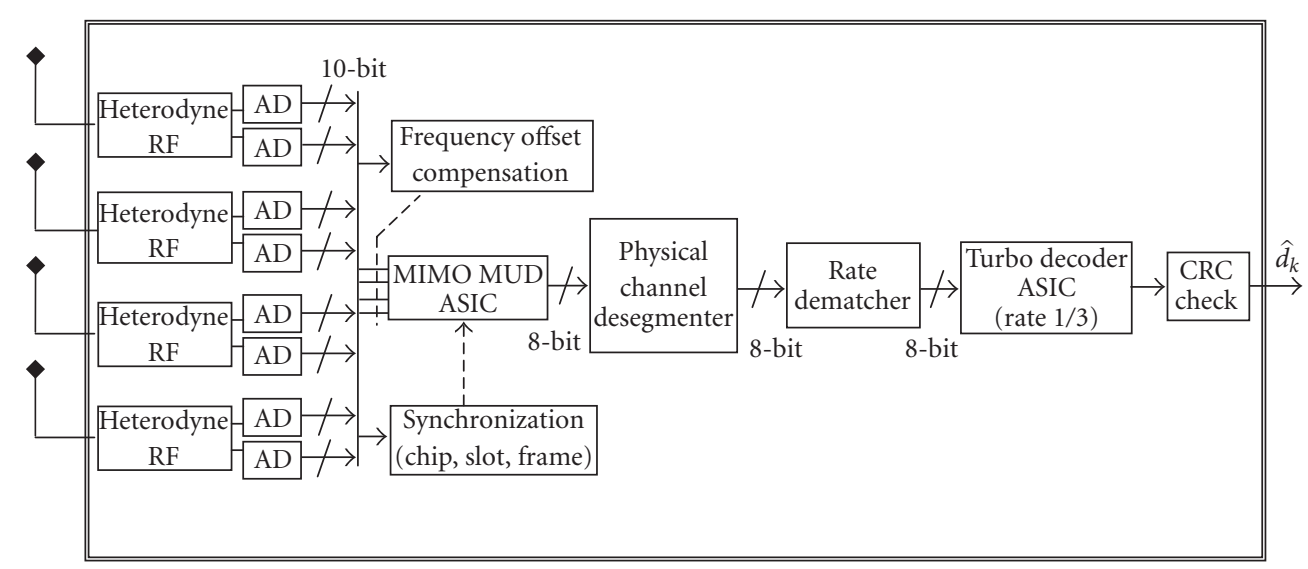

FIGURE 4: Functional block diagram of multiantenna HSDPA physical layer receiver.

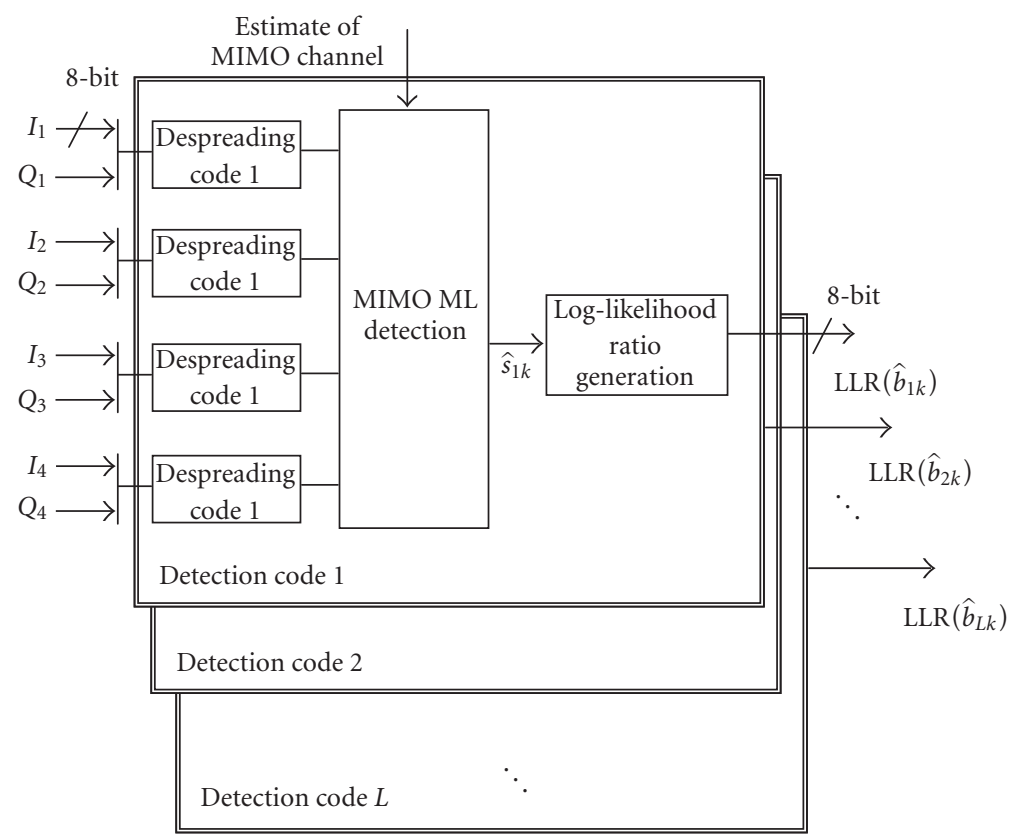

FIgURE 5: MIMO detection as implemented on the MIMO-MUD ASIC.

We measured FERs (frame error rates) with the 2 millisecond time transmission interval specified for HSDPA. Based on the FER and on the $3.84 \mathrm{MHz}$ chip rate, the throughput $T$ is obtained as

$$
T=3.84 n_{\mathrm{T}} \frac{10}{16}(1-\mathrm{FER})(\mathrm{Mbps}) .
$$

This corresponds to a system with ARQ where the frames in error are discarded.

Figure 7 presents the measured CDF (cumulative distribution) of $T$ for a transmit power of $0 \mathrm{dBm}(1 \mathrm{~mW})$ over 30 locations. We show, for $n_{\mathrm{T}}=4$ and $K=4$ terminals, each with $n_{\mathrm{R}}=1$, a comparison of SUMF and MIMO-MUD receivers. Also depicted is the throughput for $K=1$ and $n_{\mathrm{R}}=1$, for which the SUMF is optimal. Notice the large gains arising from the use of multiple-antenna transmission. It is also worth noticing the value of multiuser detection alone, which, for example, leads to a throughput increase of more than $0.5 \mathrm{Mbps}$ (at the $50 \%$ percentile point) compared to the corresponding single-user optimal transceiver. Figure 8 presents the average throughput for different transmit power levels. MIMO-MUD results, at high transmit powers, in an almost 4-fold increase in average throughput. It should be noted that a higher-order constellation could be used to combat the flooring effect shown in the figure for the single-user transceivers. However, multiuser detection would still offer some gains, as evidenced by the fact that it has superior performance even before flooring starts to occur (e.g., at $0 \mathrm{dBm}$ transmit power). Figure 9 presents the average throughput for $n_{\mathrm{R}}=1,2,3,4$ with $n_{\mathrm{T}}=4$ and with $0 \mathrm{dBm}$ (solid line) and $10 \mathrm{dBm}$ (dashed line) transmit powers. Figure 10 presents corresponding results for $n_{\mathrm{T}}=2$. In both Figures 




FIgURE 6: MIMO channel estimation as implemented on the MIMO-MUD ASIC.

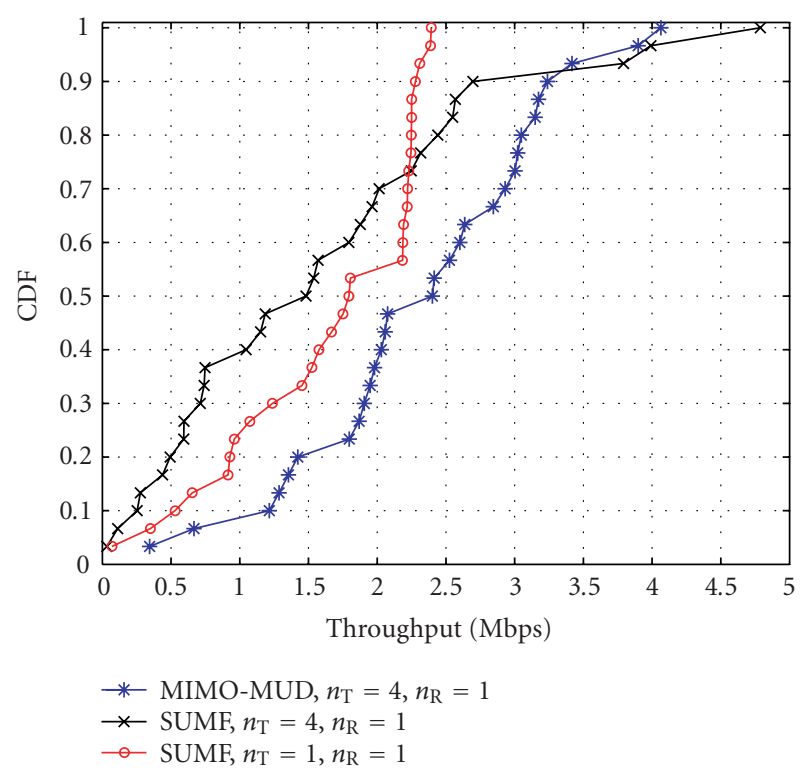

Figure 7: Measured CDF of throughput for $0 \mathrm{dBm}$ over 30 locations.

9 and 10 , we see a sizeable improvement in throughput associated with the use of MIMO-MUD, especially when $n_{\mathrm{T}}$ is larger than or comparable to $n_{\mathrm{R}}$. Although, for higher $n_{\mathrm{R}}$, the SUMF approaches the MIMO-MUD throughput, this is in part an artifact of the fact that only QPSK is used. With 16-QAM available, we expect the MIMO-MUD advantage to be largely sustained.

In order to further demonstrate the capabilities of our HSDPA MIMO prototype, we also implemented a video streaming application (using the Real-Time Streaming

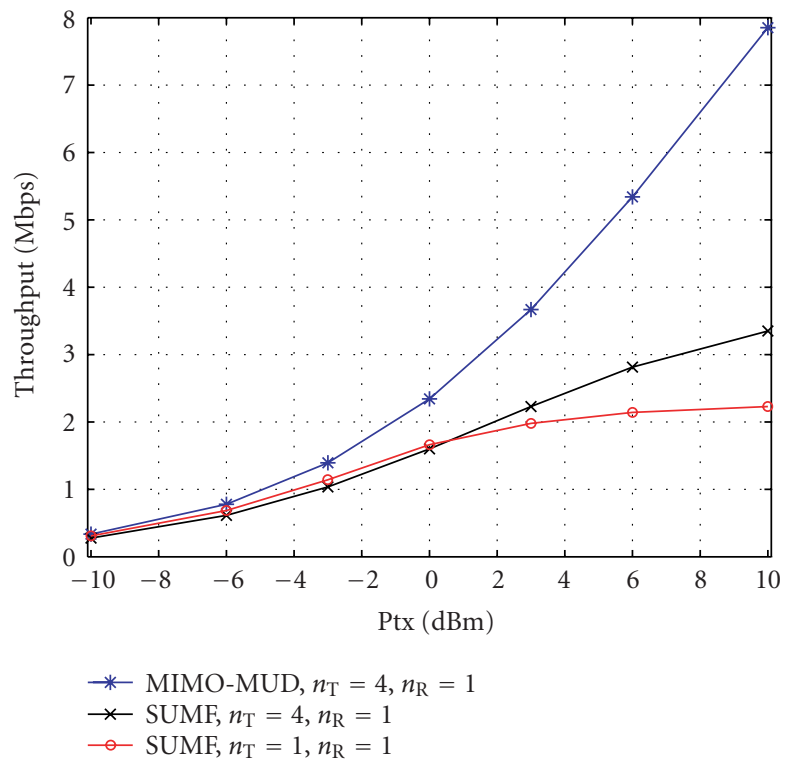

FIGURE 8: Measured average throughput versus transmit power over 30 locations.

Protocol). Video streaming rates of up to $2 \mathrm{Mbps}$ were achieved over the air (the higher layer ARQ introduced only a slight reduction in the overall throughput). In terms of interference mitigation performance, when using real-time video as each user's signal, MUD at the receiver performed very closely to the predicted behaviour and managed to separate the interfering video signals without any perceived degradation of performance as compared to each user's video stream transmitted alone. More information about these experiments can be found in [16]. 


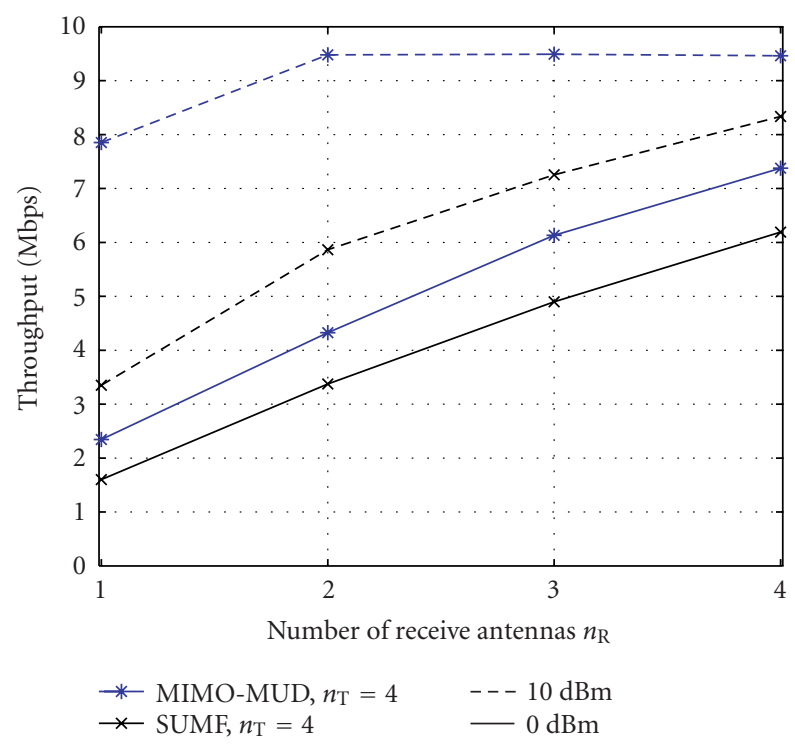

FIGURE 9: Measured average throughput versus $n_{\mathrm{R}}$ at $0 \mathrm{dBm}$ (solid line) and $10 \mathrm{dBm}$ (dashed line) over 30 locations.

\section{CONCLUSIONS}

Multiuser detection is a natural approach to signal detection in multiuser environments. Although much of the developments in this area have been motivated by CDMA, multiuser techniques are equally well suited to the spatial processing that arises with the use of MIMO, where the role of the CDMA spreading sequences is played by the fading coefficients between the various transmit and receive antennas.

In this paper, we have applied MUD to the detection of mutually interfering downlink beam transmissions aimed at different terminals. Without instantaneous channel state information at the base, these beams cannot be rendered orthogonal at the terminal receivers. Rather than simply enduring their mutual interference, we have proposed to jointly detect the signals transmitted on the intended and unintended beams.

Besides formulating such MIMO-MUD reception, we have experimentally validated the approach using a testbed that includes a commercial multiantenna base station, multiantenna terminals, and custom MIMO ASICs. The results confirm the power of MUD, especially when the number of receive antennas at each terminal does not exceed the number of transmit antennas at the base.

Besides the application that has constituted the focus of the paper, MIMO-MUD schemes carry over to other multiuser MIMO settings. If, instead of parallel beams, time/frequency multiplexing is utilized, MIMO-MUD can be applied to mitigate the impact of interference from neighboring cochannel base stations. Although, in this case, individualizing the channel estimate for each interfering base station may not always be feasible, joint detection of desired and undesired transmissions can be applied to a few dominant neighbors. Furthermore, simpler linear MMSE processing can be applied if only the aggregate interference

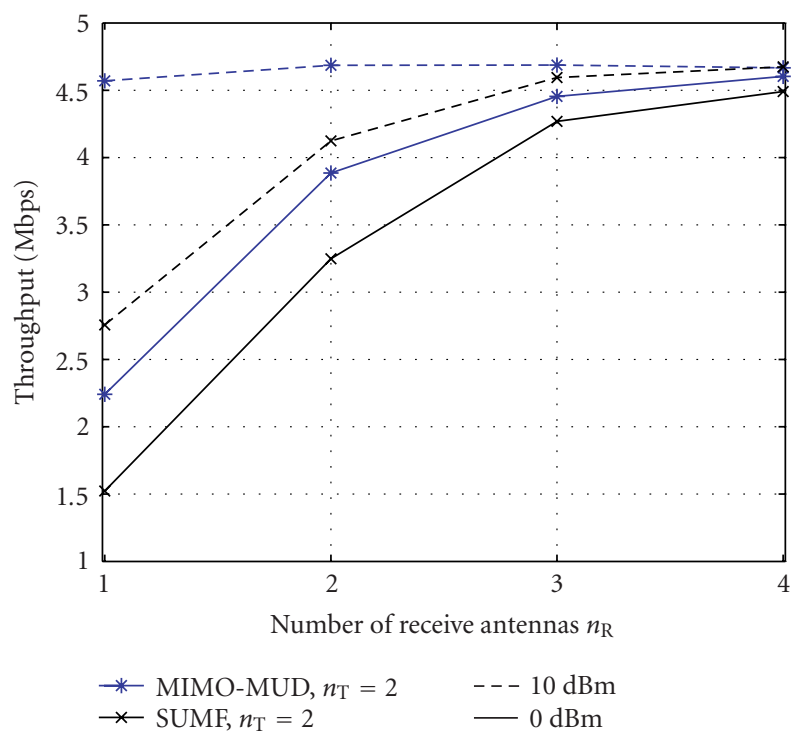

FIGURE 10: Measured average throughput versus $n_{\mathrm{R}}$ at $0 \mathrm{dBm}$ (solid line) and $10 \mathrm{dBm}$ (dashed line) over 30 locations.

covariance in (8) can be estimated. Theoretical assessments of the advantage associated with knowledge of such covariance in MIMO communication can be found in $[17,18,19]$. Actually, even before the advent of MIMO systems, earlier pioneering contributions had already demonstrated the interference suppression capability of multiple receive antennas $[10,20,21]$.

\section{ACKNOWLEDGMENTS}

The authors gratefully acknowledge the support and encouragement of many colleagues lead by Theodore Sizer, Reinaldo Valenzuela, and Stephen Wilkus, all from Lucent Technologies. The authors are particularly grateful to Peter Bosch, Sape Mullender, Susan Walker, Tran Cuong, Francis Mullany, Eric Beck, Arnold Siegel, Thomas Gvoth, and Ilya Korisch for their support. Part of this work was done under the IST project FITNESS, sponsored and funded by the FP5 European Research Framework.

\section{REFERENCES}

[1] G. J. Foschini and M. J. Gans, "On the limits of wireless communications in a fading environment when using multiple antennas," Wireless Personal Communications, no. 6, pp. 315335, 1998.

[2] I. E. Telatar, "Capacity of multi-antenna Gaussian channels," European Transactions on Telecommunications, vol. 10, pp. 585-595, 1999.

[3] W. Yu and J. M. Cioffi, "Sum capacity of a Gaussian vector broadcast channel," in Proc. IEEE International Symposium on Information Theory, pp. 498-498, Lausanne, Switzerland, June-July 2002.

[4] G. Caire and S. Shamai, "On achievable throughput of a multiantenna Gaussian broadcast channel," IEEE Trans. Inform. Theory, vol. 49, no. 7, pp. 1691-1706, 2003. 
[5] P. Viswanath and D. N. C. Tse, "Sum capacity of vector Gaussian broadcast channel and uplink-downlink duality," IEEE Trans. Inform. Theory, vol. 49, no. 8, pp. 1912-1921, 2003.

[6] S. Vishwanath, N. Jindal, and A. Goldsmith, "On the capacity of multiple input multiple output broadcast channel," in Proc. IEEE International Conference on Communications (ICC '02), vol. 3, pp. 1444-1450, New York, NY, USA, May 2002.

[7] K. I. Pedersen, P. E. Mogensen, and J. Ramiro-Moreno, "Application and performance of downlink beamforming techniques in UMTS," IEEE Commun. Mag., vol. 41, no. 10, pp. 134-143, 2003.

[8] "Beamforming enhancements," TR 25.887 v. 1.3.0, 3rd Generation Partnership Project, October 2002.

[9] S. Verdú, Multiuser Detection, Cambridge University Press, New York, NY, USA, 1998.

[10] J. H. Winters, "On the capacity of radio communication systems with diversity in a Rayleigh fading environment," IEEE J. Select. Areas Commun., vol. 5, no. 5, pp. 871-878, 1987.

[11] Q. Zhang and S. A. Kassam, "Hybrid ARQ with selective combining for fading channels," IEEE J. Select. Areas Commun, vol. 17, no. 5, pp. 867-880, 1999.

[12] A. P. Burg, E. C. Beck, D. Samardzija, et al., "Prototype experience for MIMO BLAST over third generation wireless system," IEEE J. Select. Areas Commun., vol. 21, no. 3, pp. 440451, 2003, Special Issue on MIMO Systems and Applications.

[13] D. Samardzija and N. Mandayam, "Pilot assisted estimation of MIMO fading channel response and achievable data rates," IEEE Trans. Signal Processing, vol. 51, no. 11, pp. 2882-2890, 2003, Special Issue on MIMO.

[14] D. C. Garrett, L. M. Davis, and G. K. Woodward, "19.2 Mbit/s $4 \times 4$ BLAST/MIMO detector with soft ML outputs," IEE Electronics Letters, vol. 39, no. 2, pp. 233-235, 2003.

[15] M. Bickerstaff, L. Davis, C. Thomas, D. Garrett, and C. Nicol, "A $24 \mathrm{Mb} / \mathrm{s}$ radix-4 logMAP turbo decoder for 3GPP-HSDPA mobile wireless," in Proc. IEEE International Solid-State Circuits Conference (ISSCC '03), vol. 1, pp. 150-484, San Francisco, Calif, USA, February 2003.

[16] IST-FITNESS D3.3, "Description of UMTS MTMR reconfigurability demo," www.ist-fitness.org.

[17] A. Lozano and A. M. Tulino, "Capacity of multiple-transmit multiple-receive antenna architectures," IEEE Trans. Inform. Theory, vol. 48, no. 12, pp. 3117-3128, 2002.

[18] A. Lozano, A. M. Tulino, and S. Verdú, "Multiple-antenna capacity in the low-power regime," IEEE Trans. Inform. Theory, vol. 49, no. 10, pp. 2527-2544, 2003.

[19] A. L. Moustakas, S. H. Simon, and A. M. Sengupta, "MIMO capacity through correlated channels in the presence of correlated interferers and noise: a (not so) large N analysis," IEEE Trans. Inform. Theory, vol. 49, no. 10, pp. 2545-2561, 2003.

[20] J. H. Winters, J. Salz, and R. D. Gitlin, "The impact of antenna diversity on the capacity of wireless communication systems," IEEE Trans. Commun., vol. 42, no. 2/3/4, pp. 1740-1751, 1994.

[21] J. H. Winters, "Optimum combining in digital mobile radio with cochannel interference," IEEE J. Select. Areas Commun., vol. 2, no. 4, pp. 528-539, 1984.
Dragan Samardzija was born in Kikinda, Serbia and Montenegro, in 1972. He received the B.S. degree in electrical engineering and computer science in 1996 from the University of Novi Sad, Serbia and Montenegro, and the M.S. and Ph.D. degrees in electrical engineering from Wireless Information Network Laboratory (WINLAB), Rutgers University, in 2000 and 2004, respectively. Since 2000, he has been with

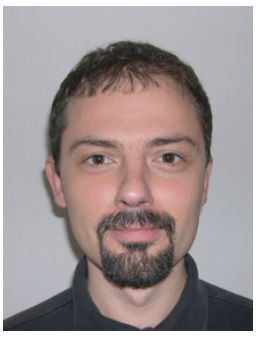
the Wireless Research Laboratory, Bell Labs, Lucent Technologies, where he is involved in research in the field of MIMO wireless systems. His research interests include detection, estimation and information theory for MIMO wireless systems, interference cancellation, and multiuser detection for multiple-access systems. He has also been focusing on implementation aspects of various communication architectures and platforms.

Angel Lozano was born in Manresa, Spain, in 1968. He received the Engineer degree in telecommunications (with honors) from the Polytechnical University of Catalonia, Barcelona, Spain, in 1992, and the M.S. and $\mathrm{Ph} . \mathrm{D}$. degrees in electrical engineering from Stanford University, Stanford, California, in 1994 and 1998, respectively. Between 1996 and 1998, he worked for Conexant Systems in San Diego, California. Since January

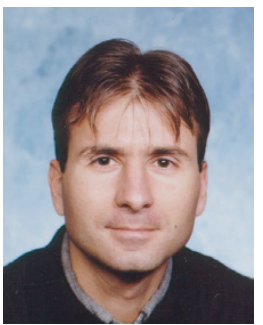
1999, he has been with Bell Laboratories, Lucent Technologies, in Holmdel, New Jersey. He has authored over 40 papers and 3 book chapters and holds 7 patents. Since October 1999, Dr. Lozano has served as an Associate Editor for IEEE Transactions on Communications.

Constantinos B. Papadias was born in Athens, Greece, in 1969. He received the Diploma of Electrical Engineering from NTUA, Athens, in 1991 and his Doctorate from ENST, Paris, in 1995. From 1992 to 1995, he was a Teaching and Research Assistant at Institut Eurécom, France. From 1995 to 1997, he was a Postdoctoral Researcher at Stanford University's Smart Antennas Research Group. Since 1997, he has been with Bell Labs' (Lucent Technologies) Wireless Research Lab, first as a member of technical staff and more recently as the Technical Manager. His current research interests lie in the areas of space-time and next-generation wireless systems. He is a Senior Member of IEEE, a Member of the Technical Chamber of Greece, and he represents Lucent Technologies at the Steering Board of the Wireless World Research Forum (WWRF). He is an Associate Editor of the IEEE Transactions on Signal Processing and he has recently received the IEEE Signal Processing Society's 2003 Young Author Best Paper Award. He is also currently an Adjunct Associate Professor at Columbia University, teaching a class on space-time wireless systems. 